\title{
Peigín Doyle, Pathway To The Cosmos: The alignment of megalithic tombs in Ireland and Atlantic Europe
}

\author{
Dublin: Wordwell Ltd., in association with National Monuments Service, \\ 2020. Paperback, fully illustrated, 143 pp. ISBN 9781916291256. €10.00.
}

\section{Carolyn Kennett}

Independent scholar

carolyn@hird.net

Pathways to the Cosmos is predominantly concerned with the alignment of megalithic tombs in Atlantic Europe, Ireland and the UK. Peigín Doyle brings together research presented by eight of the foremost researchers in the field from the successful conference of the same name held at Dublin Castle, Ireland on 15th September, 2018. The conference had been organised by Archaeology Ireland magazine on behalf of the National Monuments Service at the Department of Culture, Heritage and the Gaeltacht and the Office of Public Works in Ireland (Henty 2018, 246), which also had a direct hand in bringing about this publication.

At the core of the book is the ideal to present a holistic exploration of megalithic tombs, bringing together ideas of landscape, cosmos and megalithic culture. The 143-page colour book is beautifully illustrated throughout with high quality photographs and diagrams. It is readable, concise and organised with eight distinct chapters, all stemming from the conference lectures, and a biographical guide to the authors at the back of the book. The chapters are also enhanced by helpful standalone information boxes clearly set out from the main text by a distinct colour and conveying encyclopaedic detail without detracting from the main continuing narrative. The helpful topics in these boxes include, for example, the role of early researchers in the field from William Stukeley in the eighteenth century to Michael Hoskin in the twentieth century (pp. 16-17).

The foreword, by Malcolm Noonan, the Minister of State for Heritage and Electoral Reform, outlines the value of the publication from the perspective of the Irish Government. As a tool for highlighting the wealth of prehistoric monuments, the publication can contribute to a wider understanding of the significance of prehistoric tombs frequently found in the Irish landscape and promote the conservation of said sites. The following introduction by Gabriel Cooney builds on the foreword by illustrating the research which has been undertaken in looking at the design and alignments of passage tombs 
throughout the western European seaboard, and how collaboration of ideas is driving the field forward to a greater understanding.

Despite there being many contributing authors, the editor Doyle has clearly taken great pains to give the book an overriding voice. However, although there are clear demarcations between the topics of most chapters, there are in places overlapping content and even some contradictions. For instance, the text on page 16 argues that the method for measuring the long axis of tombs is problematic, but this method is then used to make a measurement as discussed on p. 27.

Chapter 1, titled "Sunrise Orientations and the European Megalithic Phenomenon", is based on Chris Scarre's work and gives a very condensed introduction to the historic ideas around solar orientations throughout Europe. Predominately focusing on tombs, Stonehenge is also briefly discussed. This chapter would have benefited from referencing back to original research. Suggestions that the earliest tombs in Iberia and Brittany have sunrise orientations while later tombs built in northern France and Britain have sunset orientations is a generalisation which is difficult to rationalise without links to the work which fed into this conclusion, as there are a number of famous sunrise orientations which occur in Ireland and the UK, including Newgrange and Bryn Celli Ddu. Another instance is the claim that long barrows generally point east-west or in the direction of a mid-point between the solstice positions. The choice of a picture of Pawton Quoit in a section about sunshine orientations is a little strange, as Cornish quoits have no obvious orientations. Lockyer (1906, chapters 10 and 14; 1908) did suggest some sunshine orientations at 'cromlechs' but Pawton was not included.

The second chapter is based on Fabio Silva's original research at prehistoric tombs in Iberia. A historic background to research in the region introduces the reader to the work of Michael Hoskin and the idea that such tombs were aligned to the sunrise or climbing Sun. It quickly moves into Silva's own research outlining the methodology involved in identifying orientations and his fascinating findings surrounding the red stars Aldebaran, Antares and Elnath. The chapter concludes with a wider discussion of the role that tombs may have played in the Neolithic world and of how they may have been more than funerary, holding other meanings for the societies among which they were built. A question arises from the statement that the orientation of the tombs coincides with sunrise in early February and late October, because this is curiously close to what are known as cross-quarter days. A link to this solar position, however, was discounted due to it being too cold for animal migration to summer grazing grounds. Here it would have been nice to have seen an expansion of local landscape, as on the surface the reader could surmise that at a temperate latitude and with higher temperatures than currently this may not have been the case.

The reader is introduced to Orkney in Chapter 3, and although there is a big jump in location the discussion continues in chronological order, predominantly concerned with Neolithic tombs from the early period. Jane Downes' work, which fed into this chapter, is comprehensive and at times unique. Ideas around the relationship between orientation, light from the ocean and sky and the natural landscape are creative and original. The demonstration of reverse alignments is unusual and the way people could have manipulated light is explored in a delightful way, showing the depth of research undertaken at 
the sites on Orkney. It transports the reader on a journey of imagination where they can explore the possibilities of why tombs were designed and located in the way they were, and does so in a very innovate manner.

Chapter 4, "Prehistoric Sky from 3000 BC to 500 BC", is based on Richard Bradley's extensive research in Scotland. Along with discussing landscape orientations, the chapter delves into remarkable ideas relating to the colour of the stones, to markings such as cup marks and carved rock art and to the position of stones in the landscape and what this could mean in terms of a relationship with the cosmos. It is suggested that the later part of the Bronze Age was predominantly focused on the sunset with orientations facing this way, sometimes with red stones being incorporated to reflect the Sun as it sets. Unfortunately, there is no discussion about the Moon and its role in any alignments; this had featured at the conference where Bradley felt that the Moon held importance at the later built monuments (Henty 2018). This seems to be an unfortunate oversight and there is no real discussion of possible lunar alignments throughout this book. There is also some confusing text in this chapter about the motion of the Sun which could do with further clarification. On page 56 a discussion about Clava Cairns suggests that the Cairns were constructed with a river to the southwest and an orientation to the midwinter alignment, yet it is also stated that the importance is because of "the direction in which the sun passes across the sky in summer". However, the Sun passes from northeast to northwest in the summer, so how is the southwest direction of any significance at summertime? This is not expanded upon, and seems a little odd. On page 71 there is another piece of misleading text which states that "[t]he sun travels across the sky between north-east to south-west at different times of the year". Read as written, the reader could become confused, as the Sun never travels in that direction (in the winter it travels from southeast to southwest). Otherwise, the chapter is a fascinating introduction to Scottish tombs which will leave the reader wanting to explore further.

Chapter 5 is a discussion of Frank Prendergast's comprehensive research into the orientations of every passage tomb in Ireland, and conclusions are drawn about the complexities around the interpretation of skyscapes and the cosmologies in which they are embedded. Central to this is a discussion of solar alignments and the variety of orientations found at Irish passage tombs. This is accompanied with an exploration of ideas as to why our ancestors may have made the choices they did about orientation of tombs. Dealing with a wealth of research, on the whole the chapter is presented in a concise and accessible manner. It deviates from this when it delves into ideas around astronomical significance and key differences relating to alignments at solstice positions and other times in the solar calendar. One passage in particular could have been phrased better: "Equinox, which falls midway between winter and summer solstice, would have been less easy to establish. There is no observable event in the sky to mark it, unlike the standstill of the sun at winter solstice." The Sun does not and will never "stand still"; even at the solstice it is always in motion, and the word "equinox" is a modern social construct that would have had no meaning to prehistoric people. However, although the term would have held no meaning, the midpoint Sun may have been of interest and there is evidence of mid-positions which could have fed into this idea, with the interesting alignment at 
Lough Crew Cairn T illustrated on page 83. It would have been nice to see a little more discussion of the authors' work around Knowth. The investigation into this site suggests it is unlikely that it had a mid-point orientation, but this leaves the question why two of the large Boyne Valley tombs were built with solar orientations and one was not. Should conclusions be drawn about orientations from large-scale surveys only, or can they be drawn from smaller groupings of similar sites or even individual locations? Which is more valid? Is there a place for both types of investigations? These are questions which are left unanswered at this stage in the book, although Silva in Chapter 2 provides an example of conclusions based on a single site, and it would have been interesting to see some discussion around this issue at this point.

The winter solstice at Knockroe and the work of Muiris O'Sullivan are explored next, in Chapter 6. Knockroe is an impressive passage tomb found in County Kilkenny, Ireland. It belongs to a small collection of passage tombs which focus on the locally significant hill Slievenamon, itself the location of a number of tombs and burial sites. A special relationship between locally significant hills and passage tombs is something which is seen time and time again throughout the building of megalithic tombs. The chapter gives a very good description of the tomb from its materiality (use of stone and quartz) to its construction (east tomb possibly predating the west), and the idea of a swivel stone seems unique but comparable to a movable lintel at Newgrange (see Chapter 7) and leaves the reader thinking what else could have been incorporated at sites which would be dynamic and mobile. The discussion of rock art found at Knockroe is descriptive and ideas of solar and lunar symbolism are explored along with ideas of mythology and meaning to the builders. The image of one of the decorated kerbstones under moonlight is suggestive of possible viewing opportunities and how people could have explored meaning through different channels under changing light conditions, something which has been explored further afield at Hendraburnick Quoit (Jones and Goskar 2017) and The Hurlers pavement (Nowakowski et al. 2020). The opening line may leave some readers confused: "Knockroe passage Tomb [...] is the only known passage tomb that contains two tombs aligned to two solstice events". Many readers will have visited the Boyne Valley tombs, and if they have had the opportunity to visit Knowth they will question this statement. The tomb itself is pictured within the chapter and there is a discussion about the similarities between the Boyne sites and Knockroe, particularly of rock art and colour on page 96. It would be natural to question why Knowth isn't also similarly dually aligned and why the eastern- and western-facing tombs aren't orientated for the midpoint sunrise and sunset. To work well as a continuing narrative, research about orientation at Knowth needs to have been addressed by this point of the book.

The theme of the role of art and architecture continues in Chapter 7. Beautifully illustrated throughout with images from Ken Williams, this chapter gives the reader a chance to visually appreciate the best that Irish tombs have to offer, through photographs of rock art and alignments in action. There is a discussion on how roof boxes work, with the possibility of a light-limiting quartz block being inserted at the Newgrange roof box. Following is a deeper discussion of rock art, including some of the symbolism found at the Boyne Valley tombs. It would have been nice to have delved further, in particular as 
regards solar symbolism and "Sun symbol" motifs which are found at a number of tombs. On page 114 there is a small error in the picture credits which suggests that "People witness winter solstice sunrise at Dowth passage tomb"; this is incorrect, and should read "People witness winter solstice sunset".

The final chapter is reserved for a more global perspective, based on a talk given by Clive Ruggles at the conference. It suggests that the subject of cultural astronomy and cultural heritage can benefit from approaching research in a much more inclusive and holistic manner, instead of categorising research into basic scientific disciplines. Examples from around the world of how this can be done provide an superb finale. Explored are relationships between the cosmos and the Mayan people, with complexes known to archaeologists as E-Group structures that seem to have been built predominantly for astronomical purposes. Groupings of temple monuments in Hawai'i allow for the identification of significant periods such as those which orientate to the summer solstice and then the superseding importance of the Pleiades star cluster. Finally, a discussion of the Nazca "geoglyphs" demonstrates how caution is needed when examining sites. Popular culture has linked them to astronomical events, but this has not been corroborated by recent research and although they may have had some astronomical role they were more likely designed for a variety of other purposes as well. The chapter concludes with advice on the many parallels which can be drawn with early European monuments by exploring the reasons and meanings behind sites from further afield.

This is an excellent book, which gives the reader a condensed but fascinating look at the most up-to-date understanding of megalithic tombs and their relationship to the cosmos. The book works best when directly aimed at a general reader wanting to learn more about megalithic tombs. Researchers and academics may at times find the work frustrating, though: there are no references or bibliography, and the ideas discussed leave you wanting to know more! Because of this, the text falls foul of giving sweeping statements which feel as though they have not been grounded in research, and this is where links to the original research material or further reading would have been most helpful. Within the text there are plenty of original and creative ideas of how to explore megalithic tombs and it will be interesting to see how these filter through to work undertaken in the field.

\section{References}

Henty, L., 2018. "'Pathways to the Cosmos - The Alignment of Megalithic Tombs in Ireland and Atlantic Europe'. Dublin Castle, Ireland, 15th September 2018". Journal of Skyscape Archaeology 4 (2): 246-251. https://doi.org/10.1558/jsa.37392

Jones, A. M. and T. Goskar, 2017. "Hendraburnick 'Quoit': Recording and Dating Rock Art in the West of Britain". Time and Mind 10 (3): 277-292. https://doi.org/10.1080/1751696X.2017.1341241

Lockyer, N., 1906, Stonehenge and Other British Stone Monuments Astronomically Considered. London: Macmillan.

Lockyer, N., 1908. "Some Cromlechs in North Wales". Nature 79: 9-11.

Nowakowski, J. A., C. Kennett, J. Gossip and B. Sheen, 2020. "Investigating Archaeology and Astronomy at The Hurlers, Cornwall 2013-2019". Journal of Skyscape Archaeology 6 (1): 53-85. https://doi.org/10.1558/ jsa.39523 